\title{
MECHANISMS OF PARASITE ANTIGEN UPTAKE FROM THE INTESTINE OF RATS INFECTED WITH THE NEMATODE NIPPOSTRONGYLUS BRASILIENSIS
}

\author{
MINORU YAMADA ${ }^{1}$, RYUICHI UCHIKAWA ${ }^{1}$, SHINJI MATSUDA ${ }^{2}$ \\ AND NAOKI ARIZONO ${ }^{1}$ \\ Received August 15, 2001/Accepted November 12, 2001
}

\begin{abstract}
The mechanisms of antigen-uptake at the host mucosal barrier during infection with lumen-dwelling intestinal parasites have not been well elucidated. In this study, we investigated immunolocalization of parasite excretory/secretory (ES) products in the small intestinal epithelium of rats infected with Nippostrongylus brasiliensis. Immunoelectronmicroscopy revealed intense ES immunoreactivity in the absorptive cell cytoplasm in association with lysosome-associated membrane glycoprotein (LAMP-1), and in the paracellular spaces. These results indicated that nematode antigen could be absorbed by two pathways: absorption by endocytosis and transport to lysosomes, and absorption through the paracellular pathway, suggesting altered mucosal barrier function.
\end{abstract}

Key word: Nippostrongylus brasiliensis, Rat, Intestine, Parasite antigen, Uptake

\section{INTRODUCTION}

Infection of the lumen-dwelling intestinal nematode Nippostrongylus brasiliensis induces various immune responses such as IgE antibody production and mastocytosis which are associated with the generation of Th2 cells (Matsuda et al., 1995). Along with immune reactions, N. brasiliensis infestation also induces pathological alterations in the jejunal epithelium, such as partial villus atrophy, increases in epithelial cell turnover and decreases in expression of bruch border enzymes (Hyoh et al., 1999; Perdue et al., 1989). Pathophysiological studies have also revealed changes in intestinal permeability after infection: decrease in glucose absorption rate, increased lactulose/mannitol ratio (Nolla et al., 1985; Cobden et al., 1979) and protein leakage into the intestinal lumen which results in hypoalbuminemia (Nawa, 1979; Lunn et al., 1986). Inductions of immunological responses and pathological alterations require absorption of parasite-derived molecules across intestinal epithelium, which normally have a barrier function to prevent absorption of potentially harmful materials from the intestinal lumen: uptake of macromolecules by normal absorptive cells is limited and paracellular channels are sealed by tight junctions (Anderson and Van, 1995). In this context, it is interesting to know the route of absorption of $N$. brasiliensis-derived molecules through the intestinal epithelium of infected animals. In the present study, we examined ultrastructural localization of excretory/secretory (ES) product of $N$. brasiliensis in the rat intestinal epithelium. ES product of $N$. brasiliensis comprise biologically active molecules such as acetylcholinesterase, cysteine proteinase, an interferon- $\gamma$ suppressive factor and major allergens which evoke marked IgE antibody response in the host (Yamada et al., 1991; Kamata et al., 1995; Nakazawa et al., 1995; Uchikawa et al., 2000).

\section{MATERIALS AND METHODS}

Male Spragus-Dawley rats were purchased from SLC Inc. (Shizuoka, Japan). Animals at 8 weeks of age were infected with 2,000 N. brasiliensis L3 larvae by subcutaneous injection. ES product form adult $N$. brasiliensis worms were prepared as described (Yamada et al., 1991). Polyclonal antibody against ES product was raised in Japanese white rabbits by injecting $10 \mu \mathrm{g}$ ES with Freund's complete adjuvant 3 times every 4 weeks, and the $\mathrm{IgG}$ fraction was prepared using a protein-G column (Amersham Pharmacia Biotech, Buckinghamshire, UK). Immunoblotting analyses showed that this antibody recognized multiple molecules of ES product from $17-220 \mathrm{kDa}$, but not of normal intestinal

1 Department of Medical Zoology, Kyoto Prefectural University of Medicine, Kawaramachi-Hirokoji, Kyoto 602-8566, Japan

2 Department of Parasitology, Akita University School of Medicine, Hondo-1-1-1, Akita 010-8543, Japan 
lysates of mice and rats (data not shown).

Preliminary immunohistochemical studies using paraffin-embedded tissue sections of the proximal jejunum showed that there was intense ES immunoreactivity in the villus epithelium 7 and 10 days p.i., while not in uninfected animals or those 3, 14 and 21 days p.i., suggesting that absorption of ES product is detectable during the period when a large numbers of adult worms are parasitized in the lumen. Therefore, immunoelectronmicroscopic studies were carried out 7 days p.i. using 4 rats.

For immunoelectron microscopy, segments of the jejunum approximately $20 \mathrm{~cm}$ distal to the pyloric ring were removed and fixed in a solution containing $0.5 \%$ glutaraldehyde, $4 \%$ paraformaldehyde and $0.1 \mathrm{M}$ phosphate buffer,

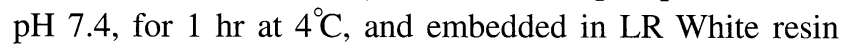
(London Resin Co., Surrey, UK) in a position so that villus structures were cut longitudinally. Ultra-thin sections were cut and incubated with $5 \%(\mathrm{w} / \mathrm{v})$ non-fat dry milk, followed by incubation with anti-ES rabbit $\operatorname{IgG}(50 \mu \mathrm{g} / \mathrm{ml})$ overnight at $4{ }^{\circ} \mathrm{C}$. For double immunostaining of ES and lysosome associated membrane glycoprotein-1 (LAMP-1), sections were further incubated with anti-LAMP-1 goat IgG $(2 \mu \mathrm{g} /$ $\mathrm{m} l$ : Santa Cruz Biotechnology, Santa Cruz, CA, USA) for 2 $\mathrm{hr}$ at room temperature. After washing, sections were incubated with $15 \mathrm{~nm}$ gold particles-conjugated goat anti-rabbit IgG (diluted to 1:20) or $5 \mathrm{~nm}$ gold particles-conjugated rabbit anti-goat $\operatorname{IgG}$ (diluted to 1:20) (BB International, Golden gate, UK) for detections of ES and LAMP-1 for 1 $\mathrm{hr}$ at room temperature, respectively, followed by staining with $2 \%$ uranyl acetate, and observed under a Hitachi $\mathrm{H}$ 7000 electron microscope.

\section{RESULTS AND DISCUSSION}

In uninfected animals, ES-specific antibody showed negligible levels of non-specific binding to absorptive cell cytoplasm, and moderate levels of nonspecific binding to microvilli (Fig. 1a). Seven days p.i., intense immunoreactivity was found on microvilli, paracellular spaces and the cytoplasm of villus absorptive cells, in which some immunoreactivity was localized to vesicular structures (Fig. 1b). Low level ES immunoreactivity was also found on the cellular membrane along the basal lamina (Fig. 1c). In a few absorptive cells, there was markedly dense immunoreactivity in the lysosome-like organelle (Fig. 1d). To determine whether ES product is transported to absorptive cell lysosomes, double immunostaining of ES and LAMP-1 was carried out. As shown in Fig. 2a, b, at least some of ES immunoreactivity showed co-localization with LAMP-1. These localizations of immunoreactivity were not observed in all villus absorptive cells, but in patchy distributions with frequencies higher in the upper part of villi. Crypt lining cells did not reveal ES immunoreactivity, while M cells over Peyer's patches showed similar distribution of ES immunoreactivity to that of villus absorptive cells (data not shown). Using nonimmune rabbit or goat $\mathrm{IgG}$, there was no gold particle deposition in paracellular spaces or in the cytoplasm of absorptive cells.

It has been presumed that only M cells over Peyer's patches are specialized for the uptake of macromolecules and antigen presentation to T cells (Mayer, 1997). However, the present studies showed that parasite-derived molecules could be transported not only through paracellular spaces but also absorbed in the villus epithelial cells, indicating that the normal barrier functions of intestinal epithelium are disrupted after infection of lumen-dwelling nematodes. The mechanism of the antigen uptake through paracellular channels is not clear from the present study. It is possible that nematode infection altered expression of adhesion molecules of villus epithelial cells. In fact, we reported previously that expression of E-cadherin, a major epithelial cell adhesion molecule, was significantly reduced at the tip of villi after $N$. brasiliensis infection (Hyoh et al., 1999). Recently, it was reported that a cysteine proteinase allergen Der pl is transported through the paracellular pathway of respiratory epithelium possibly mediated by the breakdown of tight junctions by the allergen's own proteolytic activity (Wan et al., 1999). In this respect, it is interesting that $N$. brasiliensis ES product also contains a cysteine protease (Kamata et al., 1995). Alternatively, increase of paracellular permeability might have been triggered indirectly by signals from absorptive cells, as suggested by reports that the solute permeation across tight junctions is not static and can be reversibly regulated by intracellular events with a common effector mechanism probably tied to the cytoskeleton (Madara, 1998).

The present results also showed that the ES product was absorbed in the villus epithelial cells, and at least some ES immunoreactivity was co-localized with LAMP-1, suggesting that ES product was internalized by endocytosis and transported into lysosomes. These results are consistent with the report that at least some species of macromolecules can be transported across the normal mature gut by a pinocytotic processes (Walker and Isselbacher, 1974). Since villus epithelial cells express MHC class II antigens and its expression was enhanced after $N$. brasiliensis infection (Mayrhofer and Spargo, 1990; Masson and Perdue, 1990), it is possible that some of the processed antigens are directly presented from intestinal absorptive cells to lymphocytes.

Taken together, the present studies showed that $N$. brasiliensis antigen is partly internalized in intestinal ab- 

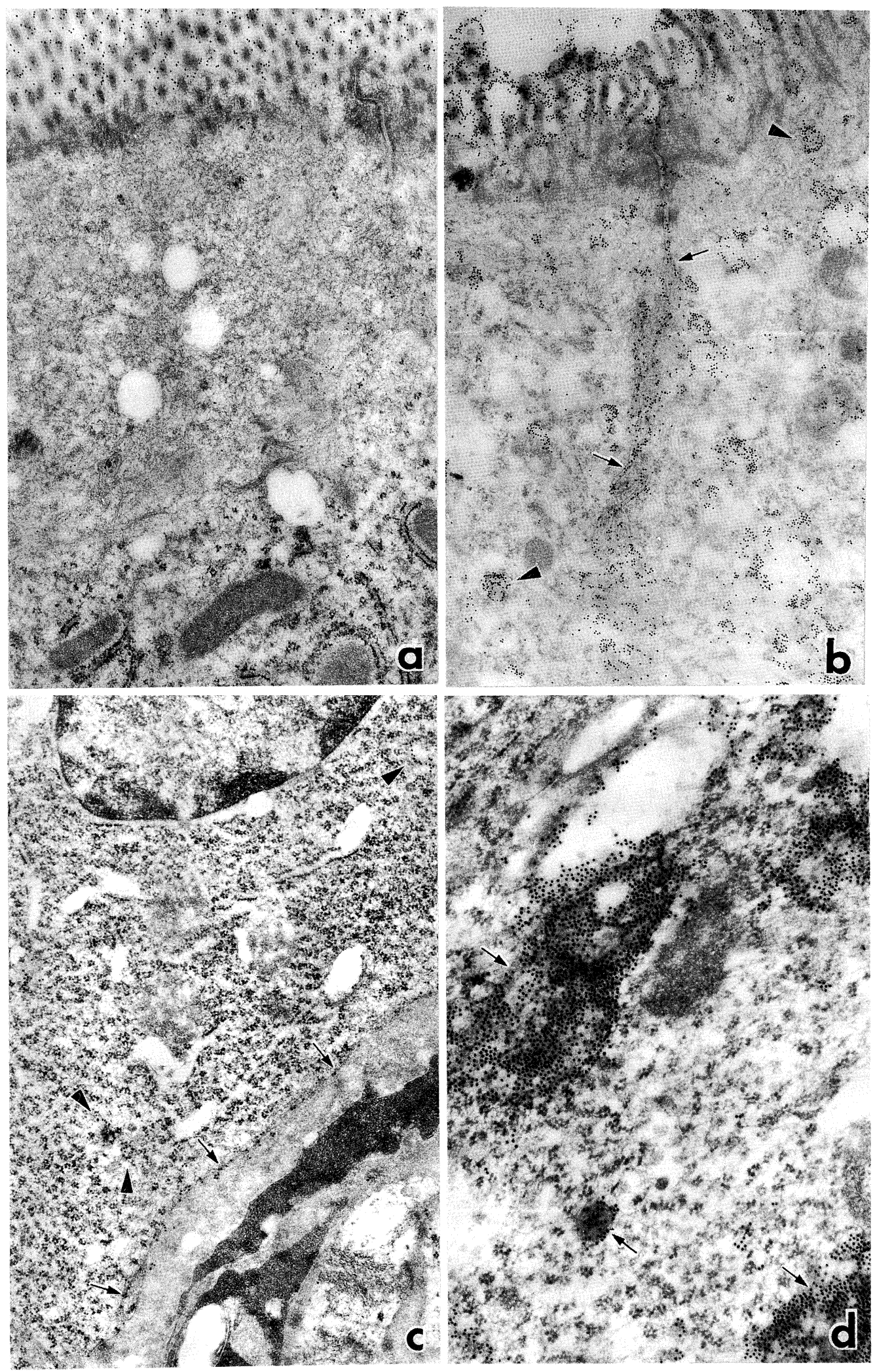

Figure 1 ES immunoelectronmicroscopy of rat small intestinal villus epithelium from uninfected rats (a) or animals 7 days after $N$. brasiliensis infection (b-d). $a$ : nonspecific gold-particle deposition is noted on microvilli, while deposition in the absorptive cell cytoplasm and paracellular spaces are negligible. $\times 20,000 . \quad b$ : Intense ES immunoreactivity is noted on microvilli, paracellular spaces (arrows), and in the cytoplasmic vesicular structures (arrowheads). $\times 20,000$. $c$ : ES immunoreactivity is observed on the basal cell membrane (arrows) and in a small vesicular structures (arrowheads) of absorptive cells. $\times 21,360$. $d$ : Intense immunoreactivity is noted in lysosome-like structures (arrows). $\times 34,860$. 

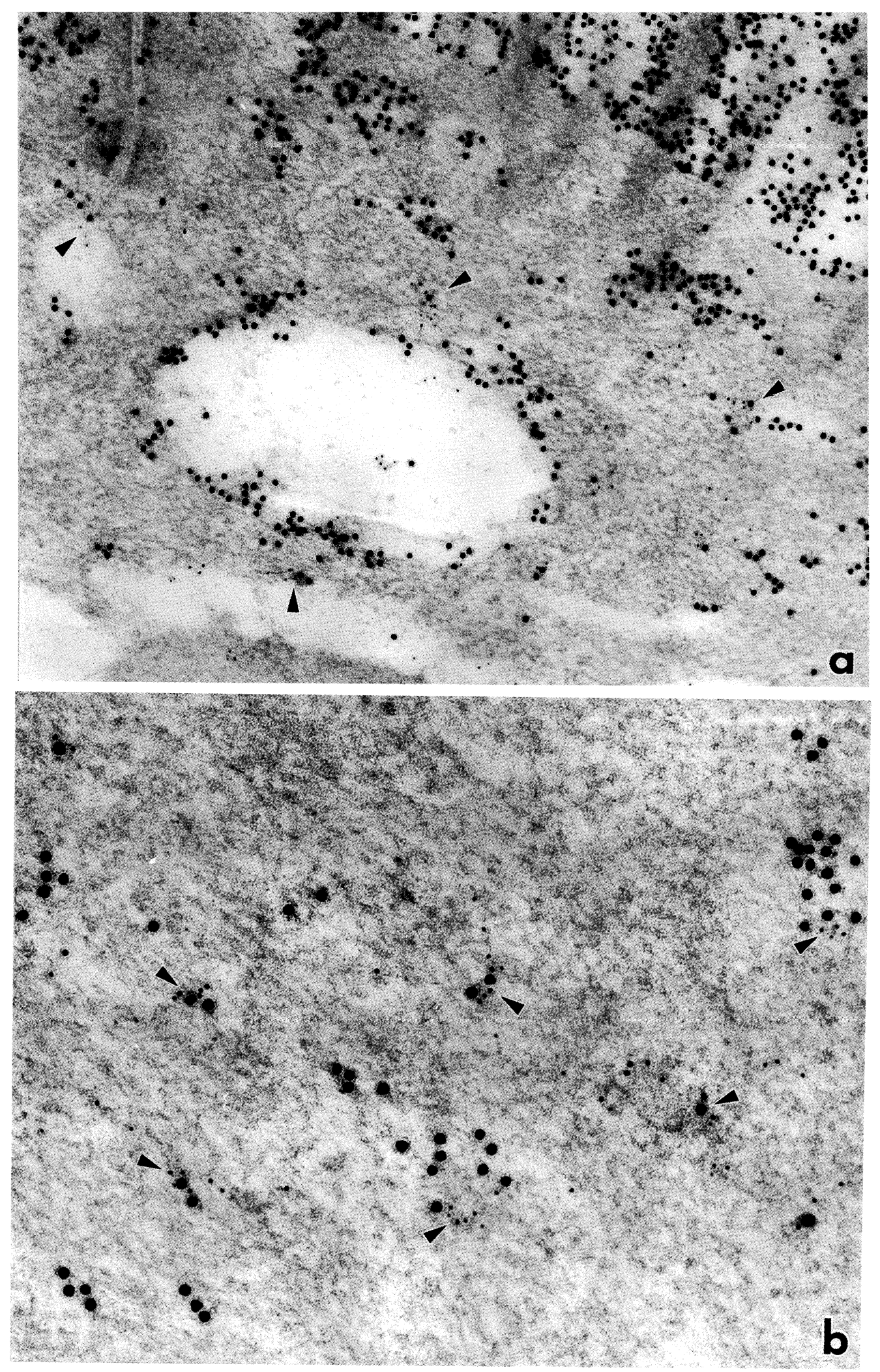

Figure 2 ES (large gold particles) and LAMP-1 (small gold particles) double immunostaining of rat small intestinal villus epithelium from rats 7 days after $N$. brasiliensis infection. ES and LAMP-1 (arrowheads) are occasionally co-localized in membrane-bound structures in the cytoplasm of absorptive cells. $a: \times 72,000 . b: \times 134,000$. 
sorptive cells and partly transported through the paracellular pathway, probably in association with the disruption of barrier function. The uptake of parasite antigen through the intestinal epithelium would have primary importance for the induction of immune reactions and development of intestinal pathology.

\section{ACKNOWLEDGEMENTS}

This study was supported by grants from the Ministry of Education, Culture, Sports, Science and Technology, Japan.

\section{REFERENCES}

1) Anderson, J.M. and Van, I.C. (1995): Tight junctions and the molecular basis for regulation of paracellular permeability. Am. J. Physiol., 269, g467-475

2) Cobden, I., Rothwell, J. and Axon, A.T. (1979): Intestinal permeability in rats infected by Nippostrongylus brasiliensis. Gut, 20, 716-721

3) Hyoh, Y., Nishida, M., Tegoshi, T., Yamada, M., Uchikawa, R., Matsuda, S. and Arizono, N. (1999): Enhancement of apoptosis with loss of cellular adherence in the villus epithelium of the small intestine after infection with the nematode Nippostrongylus brasiliensis in rats. Parasitology, 119, 199-207

4) Kamata, I., Yamada, M., Uchikawa, R., Matsuda, S. and Arizono, N. (1995): Cysteine protease of the nematode Nippostrongylus brasiliensis preferentially evokes an IgE/ IgG1 antibody response in rats. Clin. Exp. Immunol., 102, 71-77

5) Lunn, P.G., Northrop, C.A., Behrens, R.H., Martin, J. and Wainwright, M. (1986): Protein losing enteropathy associated with Nippostrongylus brasiliensis infestation and its impact on albumin homoeostasis in rats fed two levels of dietary protein. Clin. Sci., 70, 469-475

6) Madara, J.L. (1998): Regulation of the movement of solutes across tight junctions. Ann. Rev. Physiol., 60, 143159

7) Masson, S.D. and Perdue, M.H. (1990): Changes in distribution of Ia antigen on epithelium of the jejunum and ileum in rats infected with Nippostrongylus brasiliensis.
Clin Immunol. Immunopathol., 57, 83-95

8) Matsuda, S., Uchikawa, R., Yamada, M. and Arizono, N. (1995): Cytokine mRNA expression profiles in rats infected with the intestinal nematode Nippostrongylus brasiliensis. Infect. Immun., 63, 4653-4660

9) Mayer, L. (1997): Local and systemic regulation of mucosal immunity. Aliment. Pharmacol. Ther., 3, 81-85

10) Mayrhofer, G. and Spargo, L.D. (1990): Distribution of class II major histocompatibility antigens in enterocytes of the rat jejunum and their association with organelles of the endocytic pathway. Immunology, 70, 11-19

11) Nakazawa, M., Yamada, M., Uchikawa, R. and Arizono, N. (1995): Immunocytochemical localization of secretory acetylcholinesterase of the parasitic nematode Nippostrongylus brasiliensis. Cell. Tissus Res., 280, 59-64

12) Nawa, Y. (1979): Increased permeability of gut mucosa in rats infected with Nippostrongylus brasiliensis. Int. J. Parasitol., 9, 251-255

13) Nolla, H., Bristol, J.R. and Mayberry, L.F. (1985): Nippostrongylus brasiliensis: malabsorption in experimentally infected rats. Exp. Parasitol., 59, 180-184

14) Perdue, M.H., Ramage, J.K., Burget, D., Marshall, J. and Masson, S. (1989): Intestinal mucosal injury is associated with mast cell activation and leukotriene generation during Nippostrongylus-induced inflammation in the rat. Dig. Dis. Sci., 34, 724-731

15) Uchikawa, R., Matsuda, S. and Arizono, N. (2000): Suppression of gamma interferon transcription and production by nematode excretory-secretory antigen during polyclonal stimulation of rat lymph node $\mathrm{T}$ cell. Infect. Immun., 68, 6233-6239

16) Walker, W.A. and Isselbacher, K.J. (1974): Uptake and transport of macromolecules by the intestine. Possible role in clinical disorders. Gastroenterology, 67, 531-550

17) Wan, H., Winton, H.L., Soeller, C., Tovey, E.R., Gruenert, D.C., Thompson, P.J., Stewart, G.A., Taylor, G.W., Garrod, D.R., Cannell, M.B. and Robinson, C. (1999): Der pl facilitates transepithelial allergen delivery by disruption of tight junctions. J. Clin. Invest., 104, 123-133

18) Yamada, M., Nakazawa, M., Matsumoto, Y. and Arizono, N. (1991): IgE antibody production in rats against multiple components of excretory-secretory products of the nematode Nippostrongylus brasiliensis. Immunology, 72, 104-108 